\title{
Enhanced recovery strategies after penile implantation: a narrative review
}

\author{
Jeffrey L. Ellis ${ }^{1}$, Architha Sudhakar ${ }^{1}$, Jay Simhan ${ }^{1,2}$ \\ ${ }^{1}$ Department of Urology, Einstein Healthcare Network, Philadelphia, PA, USA; ${ }^{2}$ Division of Urologic Oncology, Fox Chase Cancer Center, \\ Philadelphia, PA, USA \\ Contributions: (I) Conception and design: JL Ellis, J Simhan; (II) Administrative support: J Simhan; (III) Provision of study materials or patients: \\ None; (IV) Collection and assembly of data: A Sudhakar, JL Ellis; (V) Data analysis and interpretation: None; (VI) Manuscript writing: All authors; \\ (VII) Final approval of manuscript: All authors. \\ Correspondence to: Jay Simhan, MD, FACS. Vice Chair, Department of Urology, Director of Urologic Trauma, Reconstruction and Prosthetics, \\ Einstein Healthcare Network; Associate Professor of Urology, Fox Chase Cancer Center, 1200 Tabor Road, Moss/3 Sley, Philadelphia, PA 19141, \\ USA. Email: jsimhan@gmail.com.
}

\begin{abstract}
Optimizing pain management strategies in penile implantation has historically been a challenge to urologists assuming care of patients post-operatively. In addition to the complex pathophysiology of male genital pain, the responsibility of opioid stewardship in the face of the ongoing narcotics epidemic presents its own set of challenges to experienced implanters. Recent innovations in pre- and intra-operative analgesia have provided some improvement in patient-reported pain outcomes. When used together in protocols spanning each phase of operative care, multimodal analgesia (MMA) regimens provide superior patient pain control and successfully decrease opioid usage compared to traditional opioid-based pain control. This review will systematically present literature that discusses interventions in the preoperative and intraoperative spaces aimed at optimally controlling pain. We will also highlight surgical techniques that have been demonstrated to help ameliorate post-operative pain in penile implant recipients. We will discuss the impact of MMA protocols across urology and further explore its larger impact on reducing opioid burden in the ongoing epidemic.
\end{abstract}

Keywords: Inflatable penile prosthesis (IPP); multimodal analgesia (MMA); pain management; opioid use; enhanced recovery after surgery (ERAS)

Submitted Aug 30, 2020. Accepted for publication Nov 02, 2020.

doi: $10.21037 /$ tau-20-1220

View this article at: http://dx.doi.org/10.21037/tau-20-1220

\section{Introduction}

The opioid epidemic remains a public health emergency within the United States, devastating the population and overwhelming the healthcare system. There is a welldemonstrated link between opioid addiction and postoperative opioid prescriptions. As a result, medical providers have looked to alternative methods of managing surgical pain relief. With this conceded, urology faces a unique challenge in managing post-operative pain from inflatable penile prosthesis (IPP) procedures. These procedures are becoming increasingly common and popular for their high degree of patient satisfaction. The pathophysiology of genital pain is multifactorial; as with any surgery aimed at improving quality of life, managing patient expectations is paramount. Patients sometimes visit multiple practitioners to find relief for their genital pain, which can strain the physician-patient relationship.

This review focuses on novel methods of pain management with limited opioid use in patients undergoing IPP procedures. We will review strategies for pain management in the pre-operative and intraoperative phases of care, including innovations in surgical technique to decrease post-operative pain. We will also explore 
multimodal analgesia (MMA) protocols and assess their impact on pain management paradigms in IPP surgery. We present the following article in accordance with the Narrative Review Reporting Checklist (Available at http:// dx.doi.org/10.21037/tau-20-1220).

\section{Background}

In 2014, the United States and Canada became the highest per capita opioid consumers in the world (1). As the rates of opioid prescriptions increased, so did the associated negative health consequences allowing opioid misuse to emerge as one of the leading public health crises among young and middle-aged adults. Drug overdose-related deaths have more than tripled in the past decade, and opioid-related overdoses have accounted for almost two-thirds of those deaths (2). The burden of opioid misuse, however, extends beyond these fatalities; one investigation in 2010 found that opioid dependence may be responsible for nearly 1 million disability-adjusted life-years annually in the United States alone (3).

The opioid epidemic has gained national awareness, with both state and federal governments declaring it a public health crisis. Part of the difficulty in managing this crisis stems from the popular use of opioids for postoperative pain management. Over $70 \%$ of surgical patients receive opioids following low-risk surgical procedures, with a majority of prescriptions involving either oxycodone or hydrocodone (4). In one study, $14.7 \%$ of patients at an outpatient tertiary academic medical center reported using opioids without a prescription following surgery (2). This number exceeds the national prevalence of opioid misuse, which hovers at $1.9 \%$ among US adults. Further, multiple studies have shown that opioids prescribed during or after surgery can trigger longterm use, regardless of whether patients are opioid-tolerant or have been exposed to opioids in the past (5). This has prompted medical practitioners to search for novel methods of managing pain relief to contain the scope of the epidemic.

IPP insertion is the gold standard surgery for erectile dysfunction and has been endorsed by the latest iteration of the AUA guidelines as a potential first line treatment for erectile dysfunction (6). Annual IPP implantation cases rose in the US from 17,540 in 2000 to 22,420 in 2009 , and the numbers continue to climb (7). Improvements in prosthetic design and surgical implantation techniques have resulted in longer device functional capacity and quality of life scores among patients, making this procedure popular and increasingly common (8). Still, post-operative pain continues to be a significant issue among patients undergoing IPP surgery, effecting up to $11.9 \%$ of patients at 5-year follow-up (9). Diabetes is highly co-morbid with erectile dysfunction and patients undergoing IPP surgery with uncontrolled blood glucose levels have been suggested to experience higher levels of post-operative pain and more frequent emergency room or clinic visits within 30 days of the operation (10). In an investigation into reasons for reoperation, $12.1 \%$ of IPP re-operations were found to be due to chronic pain associated with the index operation at a median re-operation time of 13.4 months (11). Half of these patients did not have an identifiable cause of their post-operative pain (i.e., contraction of the tubing, cylinder kinking), highlighting the importance of maximizing perioperative pain control (11). A single institution Mayo Clinic investigation found that IPP patients had the $4^{\text {th }}$ highest post-operative opioid prescription rate across the common urologic procedures, demonstrating a clear need for effective pain management in these patients (12).

\section{Preoperative analgesia}

The aim of preoperative analgesia is targeting the activation of the pain pathway prior to onset of surgical stimuli. Pain is often a response to tissue injury, which signals the inflammatory and nociceptive pathways and is susceptible to blockade and modulation by a variety of different mechanisms. Non-steroidal anti-inflammatory drugs (NSAIDs) are particularly effective in preoperative analgesia by inhibiting key enzymes involved in generating the initial inflammatory response. A model for the timing of preoperative NSAID administration suggests that using analgesics before surgery minimizes central stimulation of c-fibers, reducing postoperative pain intensity (13). They act by inhibiting cyclooxygenases, a group of enzymes responsible for metabolizing arachidonic acid. COX-2 is an inducible enzyme involved in pathways of pain and inflammation, while COX-1 is constitutively active in tissues. Selective inhibition of the COX-2 pathway provides analgesia without the side effect profile of non-selective COX inhibition. Parecoxib, a selective COX-2 inhibitor has been shown to be effective in managing postoperative dental pain with decreased incidence of gastroduodenal ulcers compared to nonselective COX inhibitors (14). Savitha and colleagues found NSAID administration decreased the use of intraoperative morphine in patients undergoing lumbar spinal surgery (15). In the urologic space, Mehta and colleagues studied the utility of pre-operative celecoxib 
administration on patients undergoing testicular surgery and compared it to their control group. In their cohort of 34 men, patients who received pre-operative celecoxib had significantly lower visual analog scale (VAS) scores throughout the follow-up period of 7 days and significantly less opioid consumption than the control group (16).

While NSAIDs serve to reduce pain sensitization in the peripheral pathways, another class of drugs known as gabapentinoids is aimed at targeting pain through the central pathways. Gabapentinoids include pregabalin and gabapentin, which act by binding the alpha-2 subunit of voltage-gated calcium channels in the spinal cord and brain to reduce activation. However, these drugs have a wider side effect profile than NSAIDs and are associated with higher risks for sedation and dizziness (13). Their roles in postoperative pain management thus far have been limited to small RCT studies, and an established dosing regimen is yet to be defined. Agarwal and colleagues investigated the utility of a $150 \mathrm{mg}$ dose of preoperative pregabalin in decreasing severity of postoperative pain and postoperative fentanyl requirements in patients undergoing laparoscopic cholecystectomy (17). They found that patients who received a single pre-operative dose had lover VAS scores throughout the first 24 hours post-op and had less post-operative fentanyl consumption (17). A separate investigation by Peng and colleagues studied the use of multiple low doses of pregabalin to manage pain requirements in patients undergoing the same laparoscopic cholecystectomy procedure. Patients were administered pregabalin at $50 \mathrm{mg}, 75 \mathrm{mg}$, or placebo 1 hour prior to surgery and every 12 hours after the operation for a total of 3 doses (18). Pain scoring after the procedure was significantly lower in the groups receiving multiple doses, supporting an alternative regimen compared to the one used by Agarwal's team (18). Gabapentinoids have also been examined in conjunction with NSAIDs, combining the desensitization effects of both agents. Karaca and associated investigated this combination in patients undergoing laparoscopic cholecystectomy. In their cohort, patients receiving $150 \mathrm{mg}$ pregabalin and $400 \mathrm{mg}$ IV ibuprofen prior to surgery reported lower postoperative pain scores and decreased fentanyl consumption compared to patients receiving only pregabalin (19). Additionally, the use of rescue analgesia was higher for patients taking only pregabalin (19).

Acetaminophen is another commonly used drug in preoperative analgesia. Unlike NSAIDs, acetaminophen does not actively inhibit enzymes involved in the inflammatory pathway. Instead it has a similar anti-pyretic and analgesic effect by reducing circulating prostaglandins. When combined with $600 \mathrm{mg}$ gabapentin, preoperative doses of $1,000 \mathrm{mg}$ acetaminophen have been shown to reduce postoperative pain 8 hours following the procedure and limit narcotic use in patients undergoing anorectal surgery (20). A study in 12 year old children undergoing adenotonsillectomy found that patients who received $15 \mathrm{mg} / \mathrm{kg}$ acetaminophen experienced less postoperative pain upon arrival to the post anesthetic care unit (PACU) compared to patients who received only ibuprofen or a placebo (21). Acetaminophen combined with ibuprofen has been shown to decrease postoperative opioid consumption when the combination is given in the pre-operative setting for patients undergoing orthopedic surgery (22). Preoperative administration of NSAIDs and other pain modulators have been shown to be an effective method for postoperative pain management across a spectrum of different surgical procedures.

\section{Intraoperative analgesia}

Steps taken to control pain in the operating room have been shown to have a massive impact on post-operative pain scores as well as overall patient satisfaction with the procedure. Interventions performed in the OR to maximize analgesia can be loosely categorized into two groups: application of loco-regional analgesic agents and surgical techniques designed to improve pain control. Both play a distinct role in maximizing pain control outcomes in prosthetic surgery.

There have been numerous series describing intraoperative use of local anesthesia to maximize pain control during penile prosthesis surgery. Table 1 summarizes the findings of the key studies detailing IPP intra-operative pain management strategies. Nagao and colleagues described their technique for inserting non-inflatable prosthetics while employing only a dorsal penile nerve block with $10 \mathrm{~mL}$ of bupivacaine in their series of 20 patients (33). At a mean follow-up of 3.4 years, they did not report a single case of chronic penile pain precluding their patients from engaging in intercourse (33). Ghanem and Fouad reported a series of 159 patients undergoing outpatient penile prosthetic implantation while receiving only a dorsal penile nerve block for anesthesia (23). They found that only $5 \%$ of their cohort needed additional intra-operative local anesthesia and an additional $1.8 \%$ required general anesthesia for difficulty dilating the corpora (23). They did not report any findings regarding post-operative pain 
Table 1 Summary of penile prosthetic pain management studies

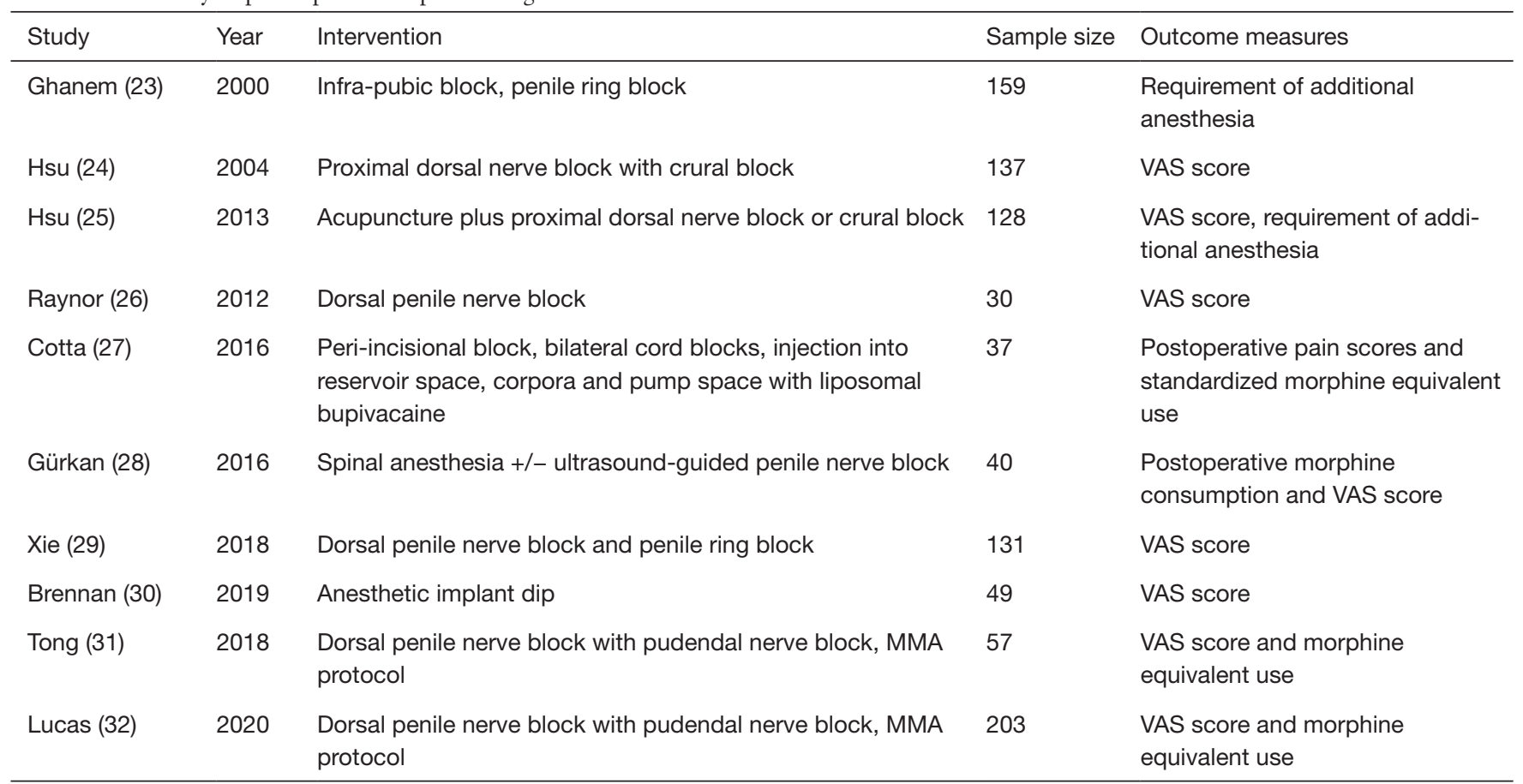

MMA, multimodal analgesia; VAS, visual analog scale.

control. While these early series' may be simple in design, they helped lay the foundation for more sophisticated investigations into ideal intra-operative anesthesia that have now become commonplace in prosthetic surgery.

Other implanters have looked at alternative types of local anesthesia to achieve better intra-operative and postoperative pain control. Hsu and colleagues studied the pudendal nerve block with dorsal penile nerve block in 21 patients and compared it to the crural block with peripenile infiltration in 137 patients undergoing both malleable and inflatable penile prosthetic surgery (24). They found that the patients receiving a crural block less frequently had post-operative pain although they required similar amounts of booster analgesia in the OR, and severity of pain as measured by post-operative VAS scores did not differ between the two groups (24). The same group reported their findings using acupuncture-assisted analgesia in various penile surgeries including prosthetic implantation in 1,481 men. Compared to other penoscrotal surgeries, patients undergoing penile implantation had the highest post-operative VAS scores and the majority of patients $(80.5 \%)$ required booster analgesia. This perhaps indicates that this technique may need some refining prior to broad implementation despite its innovation (25).
The most compelling evidence for local anesthetic use comes from studies that directly compared its implementation to a control group. Raynor and colleagues looked at a cohort of 30 men randomized to receive a dorsal penile nerve block or placebo prior to undergoing penile implantation surgery. They found that VAS scores were lowest at all assessed time intervals but patients who received a dorsal penile nerve block had significantly lower VAS scores immediately post-operatively and 4 hours subsequently thereafter (26). Cotta and colleagues did a comparative analysis investigating the use of liposomal bupivacaine in a peri-incisional and bilateral cord block versus standard bupivacaine or no local anesthesia in patients undergoing prosthesis implantation. While there was no difference between the mean overall pain scores, patients who received standard bupivacaine or no local anesthesia required significantly more morphine equivalents than patients who received the liposomal bupivacaine (27). While this shows some promise to curb opioid use, liposomal bupivacaine is substantially more expensive than the standard of care and the patients who received it had a significantly higher per patient medication cost during the investigation (\$285.54 vs. \$5.16) (27). Gürkan and associates looked at an ultrasound-guided penile nerve block for 
patients undergoing implantation using spinal anesthesia versus a control group who received no local anesthesia. Using this approach, patients who received the penile nerve block has lower VAS scores and less opioid consumption at all measured time intervals up to 24 hours (28). Xie and colleagues assessed the analgesia achieved using bupivacaine or ropivacaine in a dorsal penile nerve block plus penile ring block versus a control group receiving no local anesthesia for during implant surgery. VAS scores were assessed through post-operative day (POD) \#7. In the immediate post-operative period, VAS scores were significantly lower for patients who received any type of local anesthesia compared to the control group however, there was no difference between the group receiving bupivacaine and the cohort receiving ropivacaine (29). There was no difference among groups from POD \#2-7 in VAS scores or usage of oral opioid medication (29).

Other investigators have assessed the addition of adjunctive agents to the local anesthetic mixture to maximize pain control. Griffith and Valenzuela looked at 85 men undergoing IPP placement and administered a mixture of lidocaine, ropivacaine, sodium bicarbonate as well as dexamethasone as part of their peri-operative pudendal nerve, penile ring, and external inguinal ring block (34). All patients were discharged home without opioid pain medications and only $11 \%$ of the cohort required an opioid prescription filled after discharge (34). They report that at POD \#7-10, none of their patients were taking opioid medication (34).

Aside from different strategies of administering local anesthesia, there are several surgical techniques employed in the OR that can help minimize post-operative pain after IPP surgery. Brennan and colleagues investigated the utility of dipping the implant in ropivacaine prior to implantation in 22 men and compared post-operative pain scores to a control group of 27 men receiving a dorsal nerve block with penile ring block (30). Men in both groups had similar VAS scores in the recovery room as well as throughout follow up (30). Suarez-Sarmiento and associates assessed the effects of leaving a prosthesis inflated post-operatively on postoperative pain scores as well as surgical drain output in 91 men. They found that men with cylinders left inflated had higher post-operative phallus pain scores and while drain output was increased in patients with a deflated implant, they found no difference in infection rate or hematoma formation (35). While few other studies exist examining surgical techniques and their effect on post-operative pain control, these simple modifications seem to provide improved analgesia for implant patients.

\section{Multimodal analgesia}

The aim of MMA is to reduce postoperative pain by acting on multiple pathways modulating the pain response at different points of time. Theoretically, a combination of NSAIDS, opioids and regional nerve blocks are used block initiation and attenuation of pain signals in the nervous system (36). Surgeons have historically focused on treating pain at the downstream receptors, after the opioid receptors have already been signaled. MMA, however, uses preemptive and intraoperative analgesia to prevent central and peripheral nervous system sensitization to tissue injury (37). Compared to an approach that targets a single pathway, multimodal analgesic therapy limits the need for postoperative opioid use and prevents their undesirable side effects (37). There have been several comprehensive reviews discussing the components of MMA regimens in the anesthesia and general surgical literature however, its application in the urologic space is relatively new $(38,39)$.

The first MMA regimens described in urology were implemented outside of prosthetics. Ben-David and associates implemented an MMA protocol in patients undergoing radical retropubic prostatectomy that included pre-operative NSAID administration and intra-operative paravertebral blockade with ropivacaine (40). They compared this regimen to their standard of care comprised of wound infiltration with bupivacaine and post-operative IV ketorolac administration. In their cohort of 100 men, they found that patients who received MMA had lower VAS scores at all intervals assessed, shorter length of hospitalization, and less opioid use during their hospital course (40). Matulewicz and colleagues described an MMA protocol for patients undergoing radical cystectomy that included placement of transversus abdominus plane (TAP) catheters for continuous infusion of ropivacaine (41). They compared this to a standard of care cohort that received epidural anesthesia and found that in their cohort of 171 men, patients who received the TAP catheters had significantly lower narcotics usage in the early post-operative period (POD \#0-3) and throughout the study as well as a shorter length of stay compared to the control arm (41). Audenet and associates examined a multimodal protocol in 52 patients undergoing robotic cystectomy with extracorporeal diversion. In their protocol, patients received acetaminophen, gabapentin, and celecoxib preoperatively, a transversus abdominis or a quadratus lumborum block, and a post- 
operative regimen that included acetaminophen, gabapentin, and ketorolac and compared the protocol to patients that received a conventional, opioid-based regimen (42). The patients receiving the non-opioid regimen received a much lower dose of morphine equivalents in the hospital and had a significantly shorter length of stay without a significant difference in pain scores (42). In the endourologic space, Gridley and associates implemented an MMA protocol for patients undergoing ureteroscopy that included pre-emptive analgesia with gabapentin and acetaminophen, intraoperative NSAID and anticholinergics, and postoperative NSAID, acetaminophen, alpha-blocking medication as well as anticholinergics (43). As compared to their conventional, opioid-based cohort, they found that patients receiving MMA had less discharge opioid prescriptions and total opioid use while observing no difference in the frequency of post-operative phone calls for pain or encounters for post-operative pain (43). These protocols employed in separate urologic subspecialties demonstrate the feasibility of managing pain using a multimodal approach as compared to a conventional, opioid-based approach.

In prosthetic surgery, two investigations currently describe an MMA protocol that has promising analgesic results. In a single institution study, Tong and colleagues described a novel protocol that included preoperative NSAID, gabapentin, and acetaminophen administration, intraoperative dorsal penile nerve and pudendal nerve blockade, and a postoperative opioid-limiting analgesic regimen. When compared to a cohort receiving a conventional opioidbased regimen, the patients receiving MMA had lower VAS scores at all time intervals assessed, used fewer opioids while inpatient and on discharge, and required less opioid medication refills during follow-up (31). The same protocol was validated in a multi-institutional cohort of men undergoing IPP implantation. In this investigation, men who received MMA again had lower VAS scores in PACU and through POD \#1, required less opioids while hospitalized, and had less refills for opioid medication (32). To our knowledge, these investigations represent some of the only rigorous characterizations of pain response following penile implantation that help traverse the entire recovery period.

\section{Discussion}

Successful pain control strategies in prosthetic surgery have evolved over time with trends in pre-emptive analgesia, intraoperative techniques and finally, implementation of MMA protocols. Table 2 summarizes all of the available pharmacologic interventions that span each phase of operative care in IPP surgery aimed to reduce pain. As our knowledge deepens regarding the synergistic benefit of intervention at each phase of care, protocols become more robust. The increasing sophistication of analgesic regimens in the prosthetic arena has led to more optimal pain control and decreased opioid usage while in the hospital and following discharge. Other reviews in this space have outlined the body of literature and summarized the positive effects of improved patient pain control and satisfaction undergoing IPP placement $(44,45)$. While the benefits of reduced opioid consumption are clear, maximizing pain control and decreasing patient length of stay may have an additional benefit in our current healthcare climate. In the setting of the present COVID-19 pandemic, optimal utilization of healthcare resources is of paramount importance. Implementation of MMA protocols in other surgical fields and urologic subspecialties has been shown to decrease length of stay without adversely impacting patient satisfaction or pain control. Several studies have shown the feasibility of performing IPP surgery in an ambulatory surgery center (ASC) in appropriately selected patients $(46,47)$. Utilization of MMA protocols, particularly intraoperative components can help facilitate successful pain control such that well selected, index patients may not require hospitalization at all. Now more than ever before, healthcare resource allocation and conservation has renewed significance and can be maximized by implanters performing more IPP procedures in an outpatient in ASC setting.

\section{Conclusions}

As the opioid epidemic continues to cause significant public health morbidity and mortality, surgeons and other regular prescribers of opioid pain medication have an increasing responsibility to minimize post-operative usage. This is especially pertinent in prosthetic surgery where optimal pain control has historically been challenging and led to decreased patient satisfaction. There has been much innovation in analgesic interventions in the pre-operative and intra-operative phases of care. Recently, MMA has shown promise in improving post-operative pain control and curbing opioid medication usage in prosthetic urology. All of these interventions will help decrease the overall burden of the opioid epidemic and help allocate healthcare resource more efficaciously. 
Table 2 Pain management options for penile implantation surgery

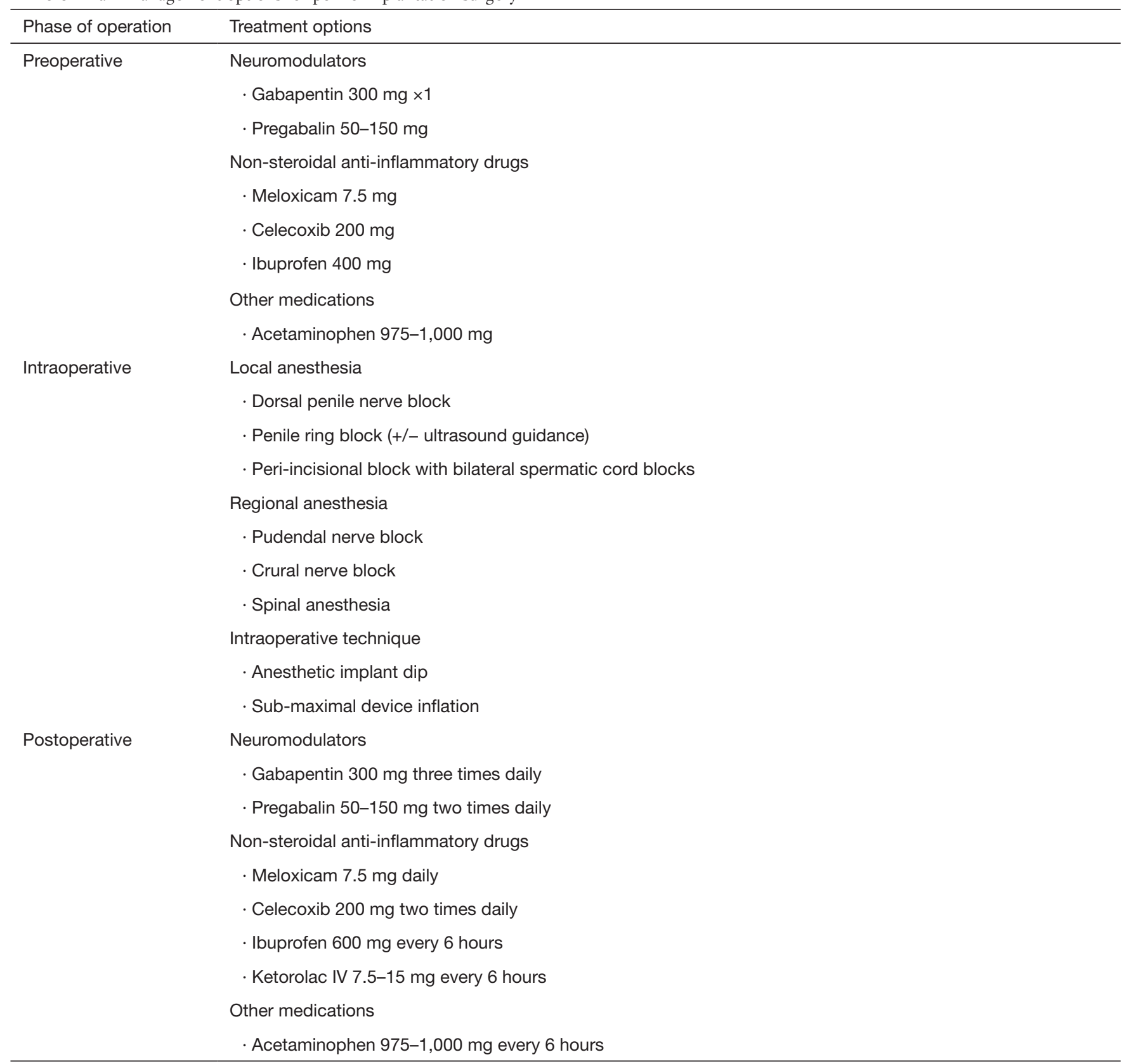

Medications must be renal-dosed in select patients.

\section{Acknowledgments}

Funding: None.

\section{Footnote}

Provenance and Peer Review: This article was commissioned by the Guest Editors (Francisco E. Martins and Tobias S.
Köhler) for the series "Controversies and Considerations of Penile Surgery" published in Translational Andrology and Urology. The article has undergone external peer review.

Reporting Checklist: The authors have completed the Narrative Review Reporting Checklist. Available at http:// dx.doi.org/10.21037/tau-20-1220 
Peer Review File: Available at http://dx.doi.org/10.21037/ tau-20-1220

Conflicts of Interest: The authors have completed the ICMJE uniform disclosure form (available at http://dx.doi. org/10.21037/tau-20-1220). The series "Controversies and Considerations of Penile Surgery" was commissioned by the editorial office without any funding or sponsorship. Dr. JS is a consultant for Boston Scientific and Coloplast. He also serves as an unpaid editorial board member of Translational Andrology and Urology from Jun 2016-Jul 2022. The authors have no other conflicts of interest to declare.

Ethical Statement: The authors are accountable for all aspects of the work in ensuring that questions related to the accuracy or integrity of any part of the work are appropriately investigated and resolved.

Open Access Statement: This is an Open Access article distributed in accordance with the Creative Commons Attribution-NonCommercial-NoDerivs 4.0 International License (CC BY-NC-ND 4.0), which permits the noncommercial replication and distribution of the article with the strict proviso that no changes or edits are made and the original work is properly cited (including links to both the formal publication through the relevant DOI and the license). See: https://creativecommons.org/licenses/by-nc-nd/4.0/.

\section{References}

1. Gomes T, Tadrous M, Mamdani MM, et al. The Burden of Opioid-Related Mortality in the United States. JAMA Netw Open 2018;1:e180217.

2. Singh GK, Kim IE Jr, Girmay M, et al. Opioid Epidemic in the United States: Empirical Trends, and A Literature Review of Social Determinants and Epidemiological, Pain Management, and Treatment Patterns. Int J MCH AIDS 2019;8:89-100.

3. Degenhardt L, Charlson F, Mathers B, et al. The global epidemiology and burden of opioid dependence: Results from the global burden of disease 2010 study. Addiction 2014;109:1320-33.

4. Wunsch H, Wijeysundera DN, Passarella MA, et al. Opioids prescribed after low-risk surgical procedures in the United States, 2004-2012. JAMA 2016;315:1654-7.

5. Hah JM, Bateman BT, Ratliff J, et al. Chronic Opioid Use after Surgery: Implications for Perioperative Management in the Face of the Opioid Epidemic. Anesth Analg
2017;125:1733-40.

6. Burnett AL, Nehra A, Breau RH, et al. Erectile Dysfunction: AUA Guideline. J Urol 2018;200:633-41.

7. Montague DK. Penile Prosthesis Implantation in the Era of Medical Treatment for Erectile Dysfunction. Urol Clin North Am 2011;38:217-25.

8. Chierigo F, Capogrosso P, Dehò F, et al. Long-Term Follow-Up After Penile Prosthesis Implantation-Survival and Quality of Life Outcomes. J Sex Med 2019;16:1827-33.

9. Jorissen C, De Bruyna H, Baten E, et al. Clinical Outcome: Patient and Partner Satisfaction after Penile Implant Surgery. Curr Urol 2019;13:94-100.

10. Reinstatler L, Rodríguez D, Goddard B, et al. Postoperative penile prosthesis pain: is it worse in diabetic patients? Int J Impot Res 2021;33:286-90.

11. Chan EP, Punjani N, Campbell JD, et al. Indications for Penile Prosthesis Revision: Lessons Learned to Limit Technical Causes of Reoperation. J Sex Med 2019;16:1444-50.

12. Ziegelmann MJ, Joseph JP, Glasgow AE, et al. Wide Variation in Opioid Prescribing After Urological Surgery in Tertiary Care Centers. Mayo Clin Proc 2019;94:262-74.

13. Kehlet H, Dahl JB. The value of "multimodal" or "balanced analgesia" in postoperative pain treatment. Anesth Analg 1993;77:1048-56.

14. Ibrahim A, Karim A, Feldman J, et al. The influence of parecoxib: A parenteral cyclooxygenase-2 specific inhibitor, on the pharmacokinetics and clinical effects of midazolam. Anesth Analg 2002;95:667-73.

15. Savitha KS, Dhanpal R, Kothari AN. The Effect of Multimodal Analgesia on Intraoperative Morphine Requirement in Lumbar Spine Surgeries. Anesth Essays Res 2017;11:397-400.

16. Mehta A, Hsiao W, King $\mathrm{P}$, et al. Perioperative Celecoxib Decreases Opioid Use in Patients Undergoing Testicular Surgery : A Randomized, Double-Blind, Placebo Controlled Trial. J Urol 2013;190:1834-8.

17. Agarwal A, Gautam S, Gupta D, et al. Evaluation of a single preoperative dose of pregabalin for attenuation of postoperative pain after laparoscopic cholecystectomy. Br J Anaesth 2008;101:700-4.

18. Peng PWH, Li C, Farcas E, et al. Use of lowdose pregabalin in patients undergoing laparoscopic cholecystectomy. Br J Anaesth 2010;105:155-61.

19. Karaca O, Pinar HU, Turk E, et al. Effects of SingleDose Preemptive Pregabalin and Intravenous Ibuprofen on Postoperative Opioid Consumption and Acute Pain after Laparoscopic Cholecystectomy. J Invest Surg 
2019;32:189-95.

20. Van Backer JT, Jordan MR, Leahy DT, et al. Preemptive analgesia decreases pain following anorectal surgery: A prospective, randomized, double-blinded, placebocontrolled trial. Dis Colon Rectum 2018;61:824-9.

21. Mahgoobifard M, Mirmesdagh Y, Imani F, et al. The analgesic efficacy of preoperative oral ibuprofen and acetaminophen in children undergoing adenotonsillectomy: A randomized clinical trial. Anesth Pain Med 2014;4:e15049.

22. Thybo KH, Hägi-Pedersen D, Dahl JB, et al. Effect of Combination of Paracetamol (Acetaminophen) and Ibuprofen vs Either Alone on Patient-Controlled Morphine Consumption in the First 24 Hours after Total Hip Arthroplasty: The PANSAID Randomized Clinical Trial. JAMA 2019;321:562-71.

23. Ghanem H, Fouad G. Penile prosthesis surgery under local penile block anaesthesia via the infrapubic space. Int J Androl 2000;23:357-9.

24. Hsu GL, Hsieh C, Wen H, et al. Outpatient penile implantation with the patient under a novel method of crural block. Int J Androl 2004;27:147-51.

25. Hsu GL, Zaid UX, Hsieh C, et al. Acupuncture assisted local anesthesia for penile surgeries. Transl Androl Urol 2013;2:291-300.

26. Raynor MC, Smith A, Vyas SN, et al. Dorsal Penile Nerve Block Prior to Inflatable Penile Prosthesis Placement: A Randomized, Placebo-Controlled Trial. J Sex Med 2012;9:2975-9.

27. Cotta BH, Welliver C, Brahmamdam A, et al. Longacting liposomal bupivacaine decreases inpatient narcotic requirements in men undergoing penile prosthesis implantation. Turk J Urol 2016;42:230-4.

28. Gürkan Y, Kuş A, Aksu C, et al. Ultrasonographyguided penile block for adult penile surgery. Can J Anesth 2016;63:780-1.

29. Xie D, Nicholson M, Azaiza M, et al. Effect of operative local anesthesia on postoperative pain outcomes of inflatable penile prosthesis : prospective comparison of two medications. Int J Impot Res 2018;30:93-6.

30. Brennan M, Suarez-Sarmiento A, Perito P, et al. 038 Analgesic Implant Soaks with Ropivacaine are as Effective as Perioperative Penile Blocks. J Sex Med 2019;16:S18.

31. Tong CMC, Lucas J, Shah A, et al. Novel Multi-Modal Analgesia Protocol Significantly Decreases Opioid Requirements in Inflatable Penile Prosthesis Patients. J Sex Med 2018;15:1187-94.

32. Lucas J, Gross M, Yafi F, et al. A Multi-institutional
Assessment of Multimodal Analgesia in Penile Implant Recipients Demonstrates Dramatic Reduction in Pain Scores and Narcotic Usage. J Sex Med 2020;17:518-25.

33. Nagao K, Ishii N, Miura K, et al. One-day penile prosthesis surgery by penile block. Int J Urol 2000;7:S56-60.

34. Griffith J, Valenzuela R. Pd22-02 Long Acting Preoperative Local Anesthetic Block To Limit Opioid Administration After Inflatable Penile Prosthesis Insertion. J Urol 2017;197:e441.

35. Suarez-Sarmiento A Jr, Brennan M, Kemper M, et al. A Prospective Evaluation of Inflatable Penile Prosthesis Left Inflated Post-Operatively. In: Submitted to AUA National Meeting; Washington DC, 2020 May 15-19.

36. Jin F, Chung F. Multimodal analgesia for postoperative pain control. J Clin Anesth 2001;13:524-39.

37. Golladay GJ, Balch KR, Dalury DF, et al. Oral Multimodal Analgesia for Total Joint Arthroplasty. J Arthroplasty 2017;32:S69-73.

38. Gritsenko K, Pain CA, Pain C, et al. Multimodal therapy in perioperative analgesia. Best Pract Res Clin Anaesthesiol 2014;28:59-79.

39. Wick EC, Grant MC, Wu CL. Postoperative Multimodal Analgesia Pain Management with Nonopioid Analgesics and Techniques: A Review. JAMA Surg 2017;152:691-7.

40. Ben-David B, Nelson JB, Chelly JE, et al. Multimodal analgesia for radical prostatectomy provides better analgesia and shortens hospital stay. J Clin Anesth 2007;19:264-8.

41. Matulewicz RS, Patel M, Jordan BJ, et al. Transversus Abdominis Plane Blockade as Part of a Multimodal Postoperative Analgesia Plan in Patients Undergoing Radical Cystectomy. Bladder Cancer 2018;4:161-7.

42. Audenet F, Attalla K, Giordano M, et al. Prospective implementation of a nonopioid protocol for patients undergoing robot-assisted radical cystectomy with extracorporeal urinary diversion. Urol Oncol 2019;37:300. e17-300.e23.

43. Gridley C, Robles J, Calvert J, et al. Enhanced Recovery After Surgery Protocol for Patients Undergoing Ureteroscopy: Prospective Evaluation of an Opioid-Free Protocol. J Endourol 2020;34:647-53.

44. Reinstatler L, Shee K, Gross MS. Pain Management in Penile Prosthetic Surgery: A Review of the Literature. Sex Med Rev 2018;6:162-9.

45. Ellis JL, Higgins AM, Simhan J. Pain management strategies in penile implantation. Asian J Androl 2020;22:34-8.

46. Segal RL, Siegelbaum MH, Lerner BD, et al. Inflatable 
Penile Prosthesis Implantation in the Ambulatory Setting: A Systematic Review. Sex Med Rev 2020;8:338-47.

47. Weinberg AC, Siegelbaum MH, Lerner BD, et al.

Cite this article as: Ellis JL, Sudhakar A, Simhan J. Enhanced recovery strategies after penile implantation: a narrative review. Transl Androl Urol 2021;10(6):2648-2657. doi: 10.21037/tau-201220
Inflatable Penile Prosthesis in the Ambulatory Surgical Setting: Outcomes From a Large Urological Group Practice. J Sex Med 2020;17:1025-32. 